\title{
PROCESSING RESONANCE SELF-SHIELDING EFFECT OF GADOLINIA BASED ON IMPTOVED SUPERCELL SCHEME
}

\author{
Jikui Li, Tiejun Zu*, Wen Yin, Liangzhi Cao \\ School of Nuclear Science and Technology, Xi'an Jiaotong University, \\ 28 West Xianning Road, Xi'an, Shaanxi 710049, China \\ *tiejun@mail.xjtu.edu.cn
}

\begin{abstract}
An improved supercell scheme has been proposed in this paper to efficiently and acc urately process resonance self-shielding effect of gadolinia. Resonance effects are cla ssified into global shadowing effect and local effects involving resonance interferenc e, spatial self-shielding effects. Two categories of effects are decoupled and treated re spectively based on different 1-D cylindrical pins. Hyperfine group method is applied to obtain multi-group cross sections for each 1-D pin. Afterwards, two categories of e ffects are coupled based on a correction formula. Because of the low efficiency for $\mathrm{C}$ arlvik method to compute collision probabilities in hyperfine group method, online ta bulation and interpolation method is developed to accelerate gaining collision probab ilities. The proposed scheme is verified against the problems of $3 \times 3$ pins with gadoli nia rod, VERA $2 \mathrm{O}$ assembly with 12 gadolinia rods and VERA 2P with 24 gadolinia rods. The numerical results suggest promising consistence of multi-group cross secti ons and eigenvalues between the proposed scheme and reference solutions.
\end{abstract}

KEYWORDS: gadolinia, improved supercell, resonance effect, collision probability

\section{INTRODUCTION}

The rapid development of computational ability allows us to perform fine neutronics calculation and have a better understanding of the detailed behavior of the whole nuclear reactor. High fidelity neutronics calculation requires accurate MG effective self-shielded XSs obtained from resonance calculation. Global-local self-shielding calculation scheme [1, 2, 3] and a method coupling Embedded Self-Shielding Method (ESSM) and hyperfine group method [4] have been proposed by the author's group to achieve highly resolved self-shielding calculations and implemented in the high fidelity neutronics calculation code NECP-X. Verification results clarified high accuracy on the uranium oxide fuel problem for both methods.

Burnable absorber-integrated guide thimbles based on boracic compositions or metallic elements like erbium or gadolinium are applied to achieve the soluble-boron-free PWR design [5]. In the new EPRTM (European Pressurized Reactor) GEN-3 reactor, Gd pins will be loaded in the first and following cores [6]. The optimized enriched Gd-155 and Gd-157 burnable poisons have been designed in a reference PWR TMI-1 core [7]. However, significant resonance self-shielding effects have appeared in gadolinia which issues a difficulty on resonance calculations. Supercell was utilized to obtain MG XSs for 
gadolinia with hyperfine group method. Nevertheless, the hyperfine group method will be timeconsuming to treat the supercell containing the gadolinia rod. Because gadolinia rods are usually divided into a dozen of regions owing to the onion-skin effect [8]. The effective self-shielded XSs should be provided for different regions. Therefore, a large number of collision probabilities need to be calculated. In order to extend the supercell scheme to gadolinia rods in the frame of the self-shielding calculation of NECP-X, the previous supercell scheme has been improved to accelerate the computation efficiency to satisfy the practical application.

In the improved supercell scheme described above, a complex supercell with fine meshes are separated into three simple configurations, and the hyperfine group method are respectively carried on these three configurations. The main reason for this treatment is that some techniques can be applied to accelerate the hyperfine group calculation, which allows the practical application of the supercell scheme. The acceleration technique used in the present work is also illustrated. Based on the valid assumption that the spatial dependence of fuel isotope number densities do not have a serious impact on the resonance shielding of the microscopic cross sections [9], the components of the sub-divided gadolinia rod in the last configuration are homogenized without any modifying geometrical mesh partition. Referring to MPACT [10] and STREAM [11] codes, a simplified interpolation tables of CPs versus a small range of macroscopic total XSs can be efficiently generated online. Generally, 100 200 cross section points are sufficient to derive an accurate interpolation tables. It should be emphasized that the interpolation and Carlvik method are combined to calculate CPs because the whole range of macroscopic total XSs is not covered in the tables. The proposed acceleration strategy named as online tabulation and interpolation method is more flexible because of eliminating the restriction in the geometrical configuration.

The aim of this paper is to treat resonance self-shielding effect of gadolinia. To achieve this goal, the derivation of the improved supercell scheme is demonstrated. Afterwards, a detailed description of online tabulation and interpolation method is provided. Numerical results are presented to verify the proposed scheme. Accuracy of results are compared with reference solution and the improved performance on efficiency is presented.

\section{DESCRIPTION OF IMPROVED SUPERCELL SCHEME}

\subsection{Construction of improved supercell}

In the improved supercell scheme, resonance effects can be classified into global shadowing effect, and local effects involving resonance interference, spatial self-shielding effects. These two categories of resonance effects are decoupled and treated individually. The shadowing effect is considered with a supercell configuration which is constructed like our previous scheme. In this supercell, adjacent nonresonant materials are homogenized to be one material to reduce computational burden with a negligible degradation in term of accuracy. Moreover, the gadolinia rod is treated as a homogeneous material, and its average XSs are obtained by hyperfine group calculations. The simplified supercell via homogenization is numbered as the No.1 pin. And then a gadolinia pin cell just consisting of gadolinia rod and moderator is constructed, ignoring the shadow effect. In this pin cell which is numbered as the No.2 pin, the gadolinia rod are also assume to be homogeneous, and its average XSs are calculated through hyperfine group method. A shadowing factor representing the shadow effect on the effective self-shielded XSs of gadolinia is obtained by ratio of the average XSs of the above two configurations. The factors can be expressed as follows:

$$
f_{x, g, k}=\frac{\sigma_{x, g, k}^{\mathrm{No} .1}}{\sigma_{x, g, k}^{\mathrm{No} .2}}
$$


where $x, g$ and $k$ mean reaction type, group number and nuclide respectively. The numerator $\sigma_{x, g, k}^{\mathrm{No} .1}$ and denominator $\sigma_{x, g, k}^{\mathrm{No} .2}$ are the average MG effective cross sections of the first and second pins. In order to treat the local effect, another gadolinia pin cell also just consisting of gadolinia rod and moderator is calculated, but in this pin cell, the gadolinia is divided into enough regions to account for the onion-skin effect. This pin cell is numbered as the No.3 pin. Through this configuration, the spatially distributed XSs ignoring the shadow effect can be obtained using hyperfine group method. The final effective selfshielded XSs of gadolinia rods in the realistic system are obtained by multiplying the spatially distributed XSs gotten from the last configuration by the shadowing factor gotten above. It should be emphasized that all annular regions within the sub-divide gadolinia rod are supposed to share the identical shadowing factors. The calculation formula is given by

$$
\sigma_{x, g, k, i}=\sigma_{x, g, k, i}^{\mathrm{No} .3} \cdot f_{x, g, k}=\sigma_{x, g, k, i}^{\mathrm{No.3}} \cdot \frac{\sigma_{x, g, k}^{\mathrm{No} .1}}{\sigma_{x, g, k}^{\mathrm{No} .2}}
$$

where $i$ is region number and $\sigma_{x, g, k, i}$ indicates the final effective self-shielded. Geometric configurations of the improved supercell are illustrated in Figure 1. Hyperfine group method is applied to resolve resonance self-shielding effect for the three pins. The efficiency of the hyperfine group method is primarily dependent on the derivation of collision probabilities for tens of thousands hyperfine groups. As presented in Figure 1, the calculation of CPs for the third pin is the most time-consuming part which still restricted the practical application of the proposed scheme. Accordingly, a newly proposed method are used to accelerate achieving CPs for the third pin in this work.
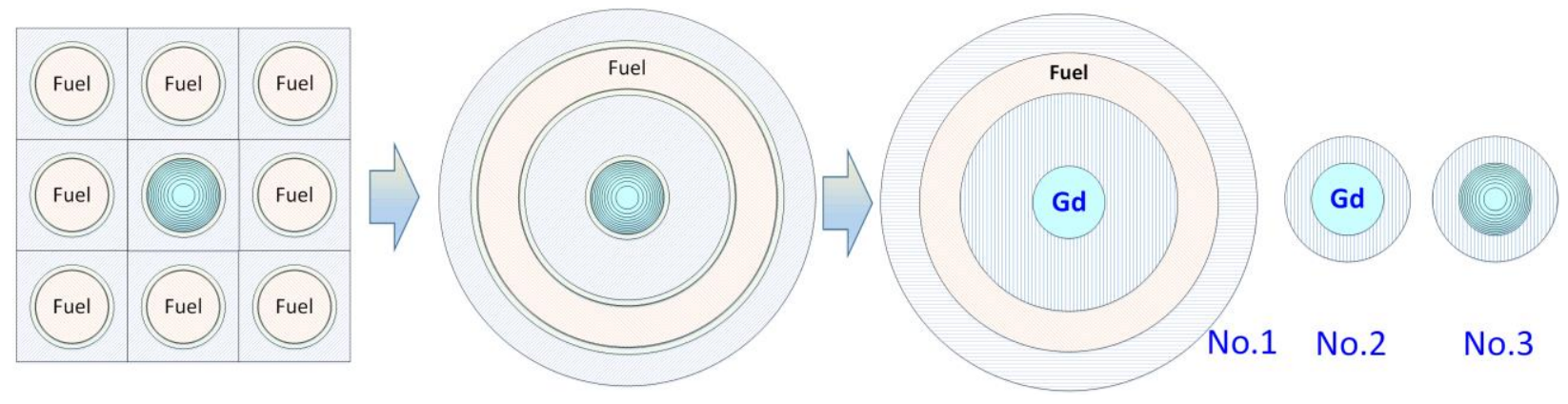

No.3

Figure 1. Geometric configurations of the improved supercell.

\subsection{Online tabulation and interpolation method}

In view of the fine mesh partition in gadolinia rod, calculation of CPs for the third pin should be further accelerated to enhance the practicability of the improved supercell scheme. Based on flat nuclide atom density distribution within the fuel rod, collision probabilities could be pre-tabulated as a function of macroscopic total XSs, so they can be calculated in advanced against several typical pin cells. This acceleration strategy has been implemented in the MPACT code [10] and STREAM code [12]. However, the tables are strictly depended on the geometrical mesh of the pin cell.

Therefore, an online tabulation and interpolation method is proposed in the present work. The atom densities within the gadolinia rod are averaged with volume weight ahead of the resonance self-shielding calculation. After getting the effective self-shielded XSs, the realistic atom densities are used for burnup calculation. In the work [9], it was proven that this treatment did not have a serious impact on the resonance self-shielding of the microscopic cross sections and isotope atom density distributions along 
the radial direction within the fuel or gadolinia rods as depletion proceeds. As regards the No.3 pin in the improved supercell scheme for gadolinia rods in VERA $2 \mathrm{O}$ problem [13], all annular regions within the gadolinia rod share the identical macroscopic hyperfine group total XSs after being averaged. The macroscopic hyperfine group total XSs are illustrated in Figure 2. It is noticed that the macroscopic total XSs of most hyperfine groups are below $5.0 \mathrm{~cm}-1$. Therefore, if the range from the minimum to maximum macroscopic total XS values is divided into several bands based on geometric sequence, a majority of the macroscopic hyperfine group total XSs are within the first band. Consequently, CPs could be tabulated online using the Carlvik method as a function of macroscopic total XSs which are within the first band. Generally, 100 200 total cross section points are sufficient to obtain a precious simplified tables. The expected collision probability tables from the $5^{\text {th }}$ to $11^{\text {th }}$ rings of the No.3 pin for gadolinia rods in VERA $2 \mathrm{O}$ is illustrated in Figure 3. When solving slowing-down equations in term of $\mathrm{CP}$, the macroscopic total XSs are confirmed whether the values are within the first band. If yes, CPs are directly gained through linearly interpolating in the tables. Otherwise, the Carlvik method would be directly utilized to generate CPs.

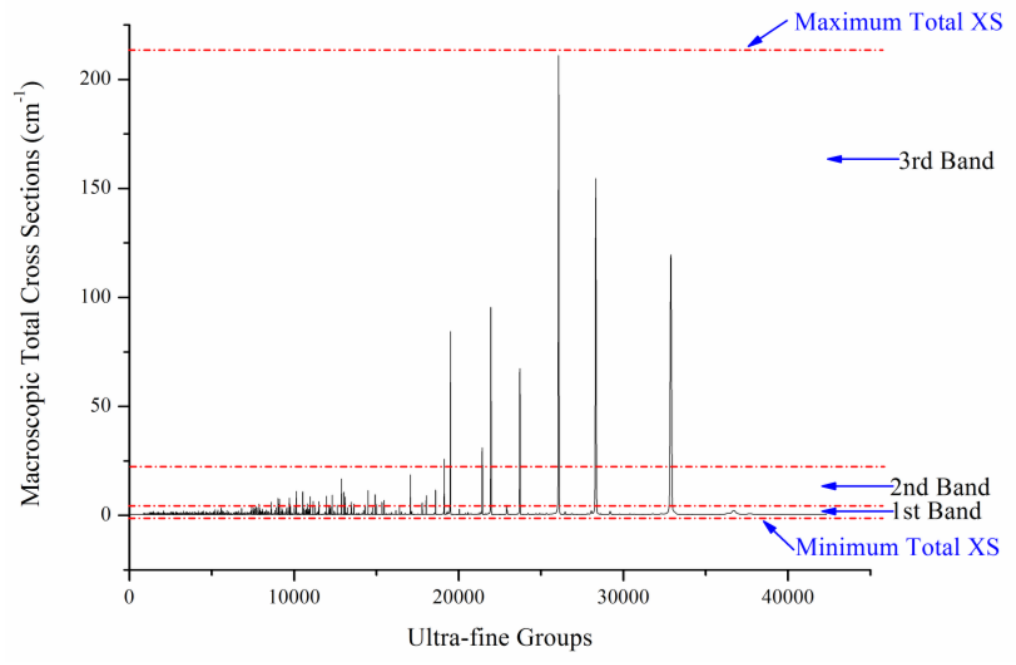

Figure 2. Macroscopic hyperfine group total cross sections of a gadolinia rod in VERA 20.

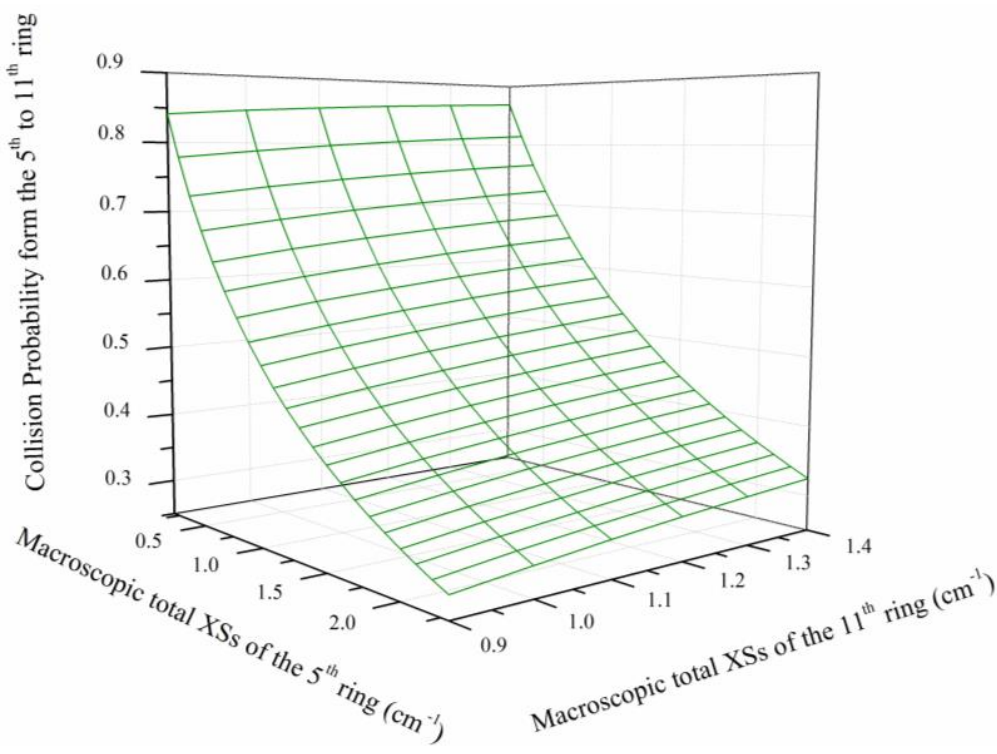


Figure 3. Tables of CPs from the $5^{\text {th }}$ to $11^{\text {th }}$ rings of the No.3 pin for the gadolinia rod in VERA $2 O$.

\section{VERIFICATION OF IMPROVED SUPERCELL SCHEME}

The improved supercell scheme has been successfully developed and utilized in the numerical reactor physics calculation code NECP-X. Global-local self-shielding calculation scheme is selected to treat resonance self-shielding effect for fuels. The verifications were based upon WIMS-69 energy group structure with the resonance energy range being extended to be from 24780 to $0.625 \mathrm{eV}$ (from 13 to 45 energy groups). Numerical results from NECP-X were based upon consistent-P2 approximation to minimize the impact of anisotropic scattering. The reference results are provided by a code system coupling Monte Carlo code [14] and burnup calculation code.

\section{1. $3 \times 3$ pins with gadolinia problem}

Input specifications containing temperatures, geometry and material compositions were completely identical with VERA 2O. Furthermore, the fuel and gadolinia rods were radially partitioned into three and ten annular regions respectively to manifest the depletion process.

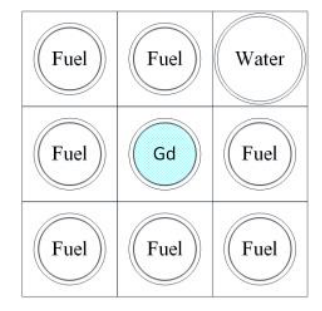

Figure 4. Configuration of the $3 \times 3$ pins with gadolinia.

Firstly, the consuming time of the improved supercell is compared with that of the previous supercell. The comparison is carried out based on the same computation platform (Intel (R) Core (TM) i7-3770 CPU @ 3.40GHz). These results in Table imply that the efficiency has been significantly improved by the proposed model. As shown in Figure 5, the maximum bias of $k_{\text {inf }}$ was less than $150 \mathrm{pcm}$ in the whole depletion process. These results in Figure 6 indicate that the representative nuclides $155 \mathrm{Gd}$ and $157 \mathrm{Gd}$ were almost depleted out after $20.0 \mathrm{GWD} / \mathrm{tU}$ since the magnitudes of atom density decreased from $1.0 \mathrm{E}$ 04 to $1.0 \mathrm{E}-07$. Therefore, the verification results of MG absorption XSs and single group absorption rates at $0.0,6.0,16.5$ and $24.0 \mathrm{GWD} / \mathrm{tU}$ are depicted in Figure 7. Relative errors of absorption XSs in most groups for ${ }^{238} \mathrm{U},{ }^{155} \mathrm{Gd},{ }^{157} \mathrm{Gd}$ and ${ }^{158} \mathrm{Gd}$ were below $3.0 \%$. Besides, the relative errors in most depletion regions for most resonant nuclides were less than $1.5 \%$ and the maximum relative errors were under $2.0 \%$ in terms of single group absorption rates.

Table 1 Comparison of efficiency between the two supercell models

\begin{tabular}{|c|c|}
\hline Model & Consuming time (s) \\
\hline Supercell & 90.247 \\
\hline Improved supercell & 1.254 \\
\hline
\end{tabular}



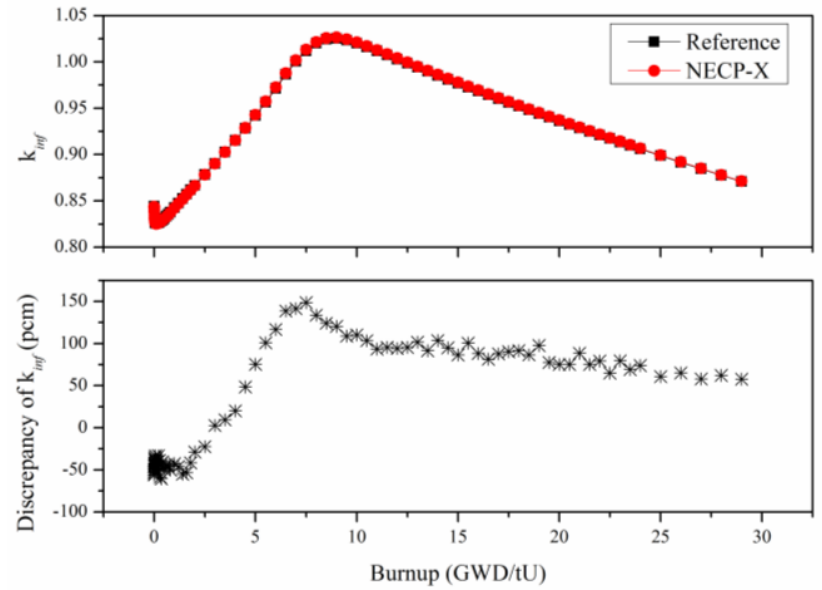

Figure 5. Comparison of $\mathbf{k}_{\text {inf }}$ for $3 \times 3$ pins with gadolinia.

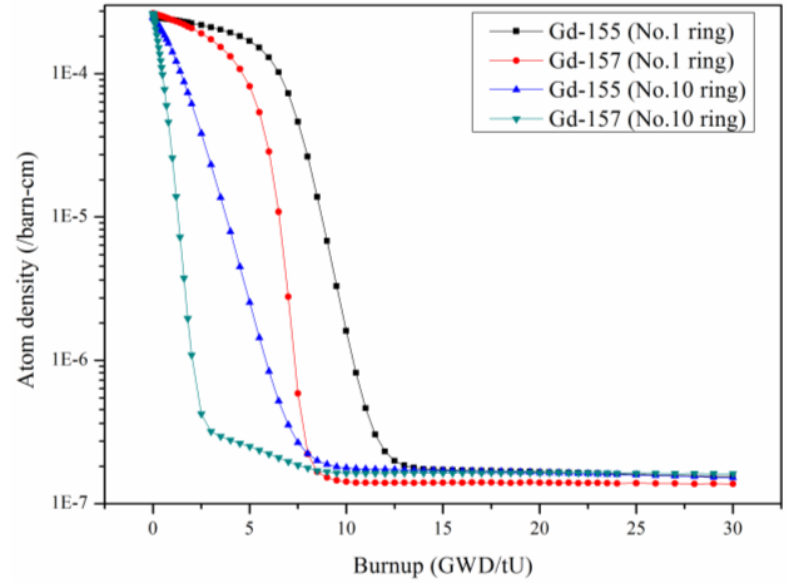

Figure 6. Change of atom densities of Gd-155 and Gd-157 with depletion.
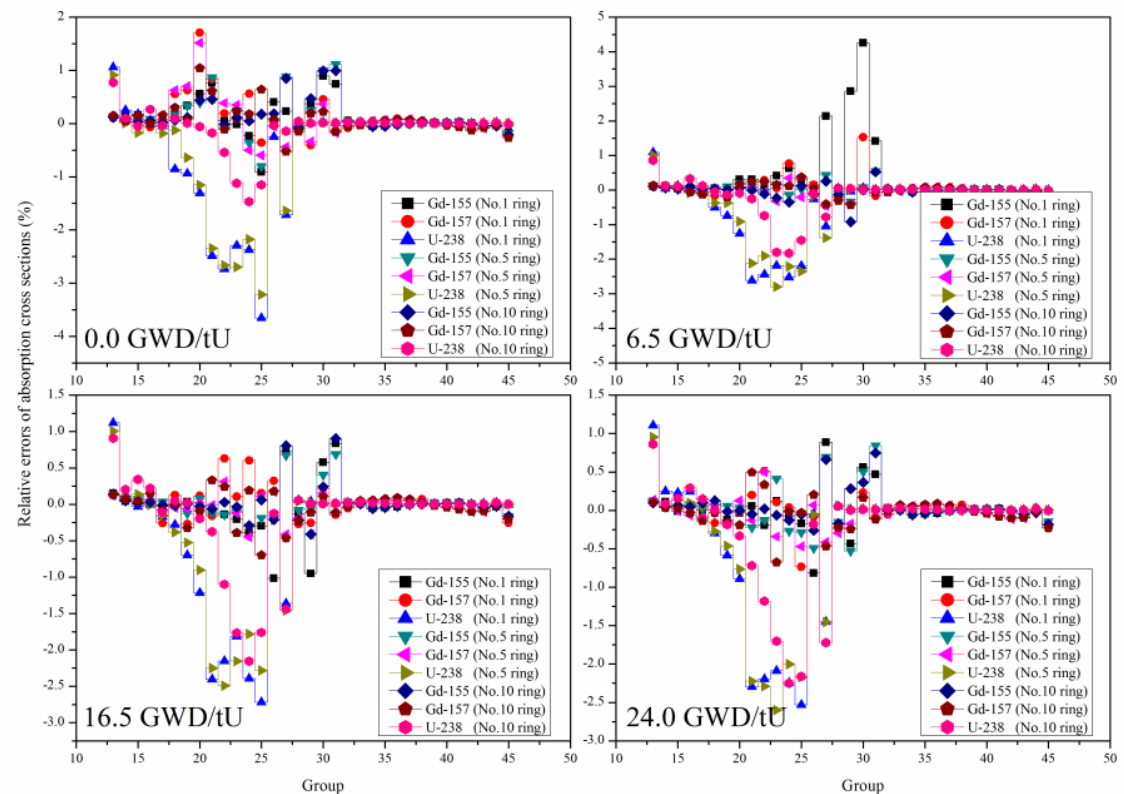
Figure 7. Relative errors of multi-group absorption XSs for the gadolinia rod.

\subsection{VERA 20}

As regards the VERA $2 \mathrm{O}$ lattice problem, the maximum discrepancy of eigenvalue in Figure 8 was below $110 \mathrm{pcm}$ in the whole depletion process.

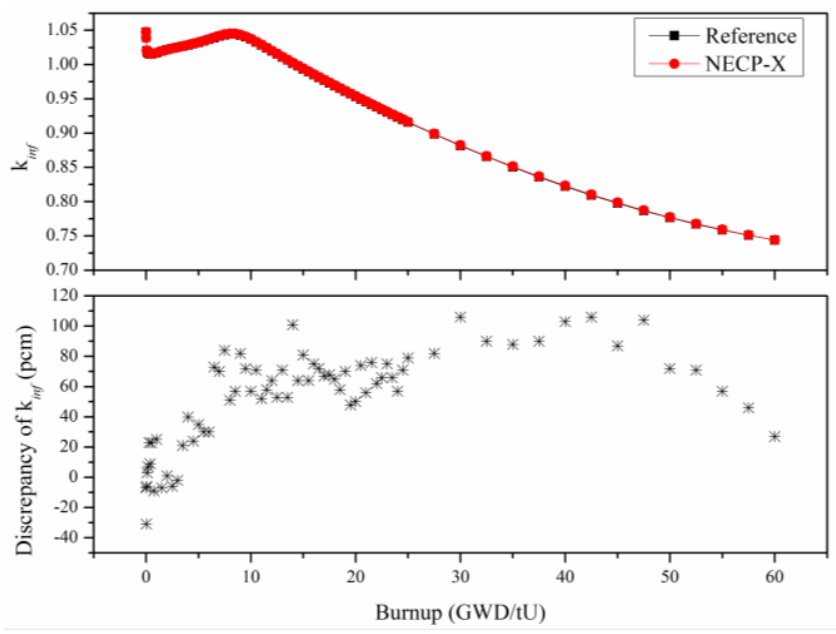

Figure 8. Comparison of $\mathrm{k}_{\text {inf }}$ for VERA 20 problem.

\subsection{VERA 2P}

As regards the VERA 2P lattice problem, the maximum discrepancy of eigenvalue in Figure 9 was below $130 \mathrm{pcm}$ in the whole depletion process. These comparison results suggest that the improved supercell scheme shows high efficiency and promising consistence with reference solutions.

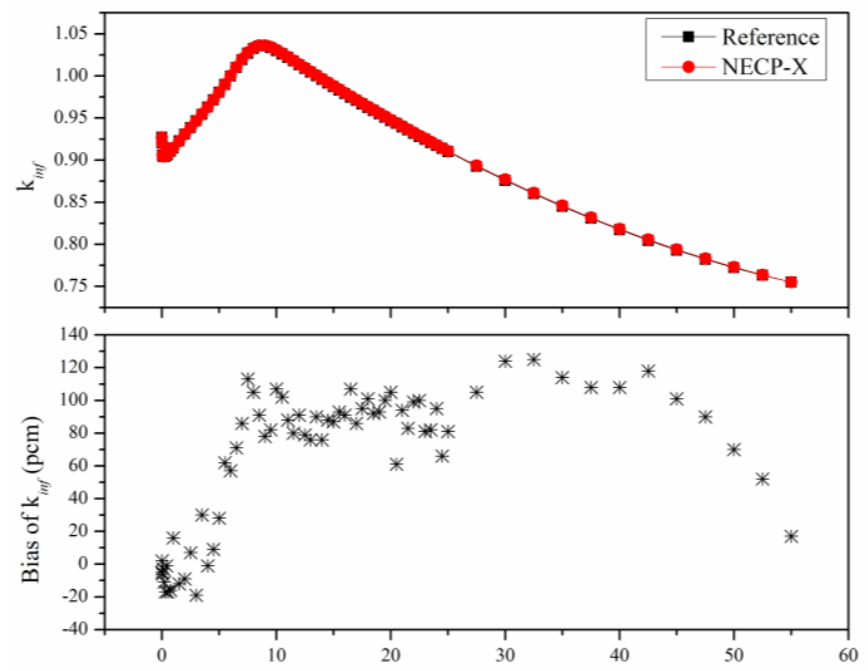

Figure 9. Comparison of $\mathbf{k}_{\text {inf }}$ for VERA 2P problem. 


\section{CONCLUSIONS}

This paper described an approach to efficiently processing resonance self-shielding effect of gadolinia rods based on improved supercell scheme. Hyperfine group method was applied to obtain multi-group effective cross sections. Compared with supercell, the proposed scheme was more efficient especially when the gadolinia rod were partitioned into a dozen of annular regions to manifest the onion-skin effect during depletions. Moreover, the online tabulation and interpolation is developed to accelerate gaining collision probabilities without compromising accuracy. The verification results based on the $3 \times 3$ pins with a gadolinia rod, VERA $2 \mathrm{O}$ and VERA 2P have demonstrated the reliability and high precision of the improved supercell scheme.

\section{ACKNOWLEDGMENTS}

This work was supported by the National Natural Science Foundation of China under Grant 11605128 and 11735011.

\section{REFERENCES}

1. Z. Liu, Q. He, T. Zu, L. Cao, H. Wu, Q. Zhang, "The pseudo-resonant-nuclide subgroup method based global-local self-shielding calculation scheme," J Nucl Sci Technol, 55, pp. 217-28 (2018).

2. Z.Y. Liu, Q.M. He, X.J. Wen, T.J. Zu, L.Z. Cao, H.C. Wu, "Improvement and optimization of the pseudo-resonant-nuclide subgroup method in NECP-X," Prog Nucl Energ, 103, pp. 60-73 (2018).

3. Q. He, Z. Liu, L. Cao, H. Wu, "Error analysis of approximations and treatments commonly made in multi-group library and self-shielding calculation based on NECP-X," Annals of Nuclear Energy, 130, pp. 418-30 (2019).

4. T.J. Zu, J.K. Li, W. Yin, L.Z. Cao, "Accurate resonance calculation method coupling simplified embedded self-shielding method and ultra-fine group method," Annals of Nuclear Energy, 120, pp. 198206 (2018).

5. M. Yahya, Y. Kim, C. Chung, "Neutronic feasibility study of a soluble boron-free PWR with the BigT burnable absorbers-15060," ICAPP 2015 Proceedings, France (2015).

6. D. Bernard, A. Santamarina, "Qualification of gadolinium burnable poison: Interpretation of MELUSINE/GEDEON-II spent fuel analysis," Annals of Nuclear Energy, 87, pp. 21-33 (2016).

7. S. Yilmaz, K. Ivanov, S. Levine, M. Mahgerefteh, "Development of enriched Gd-155 and Gd-157 burnable poison designs for a PWR core," Annals of Nuclear Energy, 33, pp. 439-45 (2006).

8. D.G. Cacuci. Handbook of Nuclear Engineering: Vol. 1: Nuclear Engineering Fundamentals; Vol. 2: Reactor Design; Vol. 3: Reactor Analysis; Vol. 4: Reactors of Generations III and IV; Vol. 5: Fuel Cycles, Decommissioning, Waste Disposal and Safeguards: Springer Science \& Business Media, (2010).

9. C.C. Stoker, Z.J. Weiss, "Spatially dependent resonance cross sections in a fuel rod," Annals of Nuclear Energy, 23, pp. 765-78 (1996).

10. T. Downar, B. Kochunas, S.M. Bowman, "Validation and Verification of the MPACT Code," (2016).

11. Y. Liu, W.R. Martin, "Pin-resolved resonance self-shielding methods in LWR direct transport calculations," Annals of Nuclear Energy, 110, pp. 1165-75 (2017).

12. S. Choi, C. Lee, D. Lee, "Resonance treatment using pin-based pointwise energy slowing-down method," Journal of Computational Physics, 330, pp. 134-55 (2017).

13. A.T. Godfrey, "VERA core physics benchmark progression problem specifications," (2014).

14. P.K. Romano, N.E. Horelik, B.R. Herman, A.G. Nelson, B. Forget, K. Smith, "OpenMC: A state-ofthe-art Monte Carlo code for research and development," Annals of Nuclear Energy, 82, pp. 90-7 (2015). 